\title{
¿Contar o callar?: La respuesta de los adolescentes del sistema de justicia juvenil ante las experiencias de exposición indirecta a violencia
}

\section{Silencing or telling?: The response of adolescents in juvenile justice setting to indirect violence exposure}

\author{
Recibido el 3 noviembre 2016/Publicado el 5 abril 2017
}

\begin{abstract}
Graciela Susanne ${ }^{1}$, Noemí Pereda, y Georgina Guilera
Grupo de investigación en Victimización Infantil y Adolescente (GReVIA)

Universidad de Barcelona
\end{abstract}

\begin{abstract}
RESUMEN
El objetivo del estudio ha sido evaluar las múltiples experiencias de exposición indirecta a violencia (EIV) de los adolescentes infractores, analizar si callan o cuentan lo que han experimentado a lo largo de sus vidas y quiénes son sus interlocutores. La muestra consta de 101 adolescentes de entre 14 y 17 años, en cumplimiento de medidas en centros de justicia juvenil de Cataluña. La EIV fue evaluada con el Juvenile Victimization Questionnaire (JVQ; Finkelhor et al., 2005), versión autoinforme. Los resultados indican que prevalece la cultura del silencio. En el ámbito familiar se observan tasas de silencio del $75 \%$ en la violencia de padres a hijos y de los padres entre sí del 69\%; y en el comunitario del $57.5 \%$ en agresiones sin arma y del $51 \%$ en asesinato de familiar o amigo. Los interlocutores más frecuentes son los amigos, a quienes les cuentan experiencias de disturbios callejeros o tiroteos (45.2\%) y agresiones con $\operatorname{armas}(42 \%)$.
\end{abstract}

\footnotetext{
${ }^{1}$ La correspondencia debe dirigirse a: Graciela Susanne. Sección de Psicología Clínica y Psicobiología. Facultad de Psicología, Universidad de Barcelona. Passeig Vall d'Hebron, 171. C.P. 08035 Barcelona. mgsusanne@ub.edu
} 
Palabras clave: Testigos de violencia; justicia juvenil; victimización; violencia; España.

\begin{abstract}
The goal of the study is to give visibility to the multiple experiences of indirect exposure to violence (EIV) to which young delinquents are exposed during their lives, to observe whether they keep silent or tell somebody about them, and who are their most frequent interlocutors. The sample consisted of 101 youth aged between 14 and 17 years, mainly recruited from detention centers (77.2\%) in Catalonia, Spain. EIV was assessed with the Juvenile Victimization Questionnaire (JVQ; Finkelhor et al., 2005), self-report version. Results show that keeping silent is the prevalent behavior among them, with highest rates of silencing within the family context (violence from parents to siblings, $75 \%$, and between parents, 69\%). With highest rates within the community context, by assaults without weapon (57.5\%), and murder of relative or friend $(51 \%)$. Friends are the most frequent interlocutors by community violence experiences (riots and shootings, $45.2 \%$, and assaults with weapons, $42 \%$ ).
\end{abstract}

Keywords: Witnessed violence; juvenile delinquency; victimization; violence; Spain.

\title{
1. Introducción
}

Los adolescentes se encuentran expuestos a grandes dosis de violencia, y no sólo en videojuegos y películas, como se suele creer, sino además y principalmente, en la vida real (Mrug et al., 2015). Estas experiencias se dan en el ámbito familiar o en su entorno social y suelen quedar ocultas y silenciadas, invisibles a los ojos de los adultos y de la sociedad en general (UNICEF, 2014; Unnever y Cornell, 2004). Se define la "exposición indirecta a violencia" (EIV) o ser testigo de violencia, en sentido amplio e inclusivo (Holden, 2003), como las experiencias en las que la víctima es testigo presencial (ve, oye), toma conocimiento o se ve implicada en episodios reales (no ficticios) de violencia ejercida sobre otra persona o grupo, quedando excluida la visualización de imágenes de violencia (cine, videojuegos, televisión).

En el ámbito científico, la EIV se ha asociado con el desarrollo de conductas delictivas, antisociales y agresivas en múltiples estudios empíricos (Barker et al., 2008; Brookmeyer, Henrich y Schwab-Stone, 2005; Cisler et al., 2012; Covey, Menard y Franzese, 2013; Egeland, 2010; Huang et al., 2015; Loeber y Stouthamer-Loeber, 1998; McCart et al., 2007; Menard, Covey y Franzese, 2015; Reckdenwald, Mancini y 
Beauregard, 2013; Thornberry y Krohn, 2004; Widom, 1989) y también en rigurosos metanálisis (Evans, Davies y DiLillo, 2008; Kitzmann et al., 2003; Wolfe et al., 2003).

La EIV aumenta el riesgo de desarrollar comportamientos violentos en tanto proporciona los modelos conductuales que facilitan el aprendizaje de la violencia (Akers, 1985; Avakame, 1998; Bandura, 1977; Loeber y Stouthamer-Loeber, 1998) como herramienta para la resolución de los conflictos interpersonales (Besemer, 2011; Black, Sussman y Unger, 2010), y de la delincuencia como estilo de vida (Eitle y Turner, 2002; Erdelja et al., 2013; Herrera y McCloskey, 2001). En este sentido, la Organización Mundial de la Salud (World Health Organisation; WHO, 2013) ha alertado sobre el riesgo que comporta la EIV para el desarrollo infantojuvenil, teniendo en cuenta que los niños, niñas y adolescentes expuestos podrían llegar a ser ellos mismos en el futuro perpetradores o, nuevamente, víctimas de violencia. Además, ha denunciado la tendencia social a demonizar a los jóvenes infractores y a ignorar, en cambio, sus experiencias de victimización. Por todo esto, insta a implementar estudios centrados en sus historias de victimización a lo largo de la vida (life-course approach), y a revertir la destinación de esfuerzos y presupuestos de justicia juvenil hacia la prevención y atención de estos jóvenes (WHO, 2010; 2013; 2014).

Una cuestión a tener en cuenta es que, si bien no todos los jóvenes expuestos a violencia desarrollan conductas delictivas (Cuevas, Turner y Ormrod, 2007), ni todos los delincuentes han sido previamente expuestos a violencia (Finkelhor, 2007), la EIV se ha identificado como la forma de victimización más frecuente entre los adolescentes ingresados en el sistema de justicia juvenil, con tasas de prevalencia que oscilan entre el 74.1\% (Abram et al., 2014) y el 97\% (Pereda, Abad y Guilera, 2015). Estas cifras son mucho más altas que las halladas en otros tipos de muestras de jóvenes de edades similares (Abram et al., 2013; Cyr et al., 2013; DeHart y Moran, 2015), situando a los jóvenes infractores entre los segmentos de la población más expuestos a episodios de violencia.

La invisibilidad de las experiencias de EIV antes mencionada, también se detecta en la literatura criminológica, en tanto que, tradicionalmente, los estudios se han centrado en las carreras delictivas de los jóvenes (Forcadell et al., 2004; Mancho, 2012; Martínez-Catena y Redondo, 2013; Van Domburgh, et al., 2009), o en su perfil sociodemográfico o psicológico (Martín Solbes, 2008; Oriol Granado, 2013; Rechea, 
Fernández y Cuervo, 2008), siendo muy escasos los que abordan sus experiencias de victimización. A pesar de ello, en los últimos años se están produciendo cambios que se observan en el creciente interés por parte de los investigadores en estudiar los tipos de exposición a violencia y la conducta de las víctimas ante ellas.

Estudios recientes indican que, si bien algunas víctimas y testigos cuentan a otras personas las experiencias de victimización en busca de ayuda o solución para el conflicto, o para entender mejor la situación vivida (Unnever y Cornell, 2004), en general, son reticentes a contar sus experiencias de victimización (Bauman et al., 2016; Dijkstra, Lindenberg y Veenstra, 2008; Hallsworth y Young, 2008; Newman, Murray y Lussier, 2001; Oldenburg et al., 2016; Smith y Shu, 2000; Unnever y Cornell, 2004), y mantenerse en silencio es la respuesta más frecuente ante la exposición a violencia. El silencio de las víctimas ha sido relacionado con mecanismos psicológicos complejos, tales como la represión, el trauma, los procesos de memoria (el olvido) (Pasupathi y McLean, 2010; Sorsoli, 2010) y la desensibilización a la violencia (McCart et al., 2007); factores emocionales como, por ejemplo, el miedo (Hallsworth y Young, 2008; Kiewitz, et al., 2016), la apatía o el desinterés (Hektner y Swenson, 2012; Smith y Shu, 2000); y factores cognitivos tales como el riesgo percibido y el análisis de costes y beneficios (Frattaroli, 2006; Unnever y Cornell, 2004). A este silencio se suma el de los jóvenes testigos, espectadores u observadores (witnesses, bystanders, observers) de esos episodios que, a su vez, deciden callar, fomentando así la llamada "cultura del silencio" (Smith y Shu, 2000) y contribuyendo a incrementar la invisibilidad de las experiencias de victimización.

Según la literatura, se guarda silencio por "no encontrar las palabras" o "quedarse sin palabras" debido al miedo, estrés o alta emotividad que provoca el evento al que se ha estado expuesto (Berger, 2004), o por no tener suficiente habilidad para construir una narración coherente e inteligible de los sucesos (Bohanek, Fivush y Walker, 2005). Asimismo, puede ser una decisión estratégica, a menudo motivada por la presión de fuerzas externas, tales como obediencia o lealtad al grupo al que se pertenece, a la autoridad o poder de alguien, o a las normas sociales (Fivush, 2010), sin olvidar que también se guarda silencio por olvido, negación o vergüenza, por lealtad individual al victimario, o por creer que son las víctimas directas quienes han de atender 
sus asuntos sin intervención de otros (Hektner y Swenson, 2012; Oldenburg et al., 2015).

Disponer de interlocutores a quienes dirigirse, como padres y familiares (Pasupathi y Hoyt, 2009; Rousseau et al., 2014), maestros y profesores (Oldenburg et al., 2015; Reitsema y Grietens, 2015; Unnever y Cornell, 2004) y, particularmente en la adolescencia, de amigos, personas clave para el desarrollo de la identidad (Pasupathi y Hoyt, 2009), facilita que los jóvenes opten por contar estas experiencias. La respuesta de los adultos (falta de reactividad, apatía o impasividad ante la victimización) y de las instituciones informa a los jóvenes acerca de la aceptación que tienen la violencia y la victimización como parte de la cultura o contexto en el que viven (Reitsema y Grietens, 2015; Unnever y Cornell, 2004), y también acerca de la conveniencia de contar o callar sus experiencias según en qué entorno y ante qué persona se encuentran. Así, si a pesar de tener a quien dirigirse, las respuestas de los interlocutores conllevan menosprecio, acusaciones, amenazas, o no dan crédito a lo que los jóvenes les cuentan (Kaufmann, et al.; Lens, et al., 2016; Staller y Nelson-Gardell, 2005), éstos no actuarían como factores facilitadores sino como inhibidores de la conducta de contar.

Contar lo que se ha visto, oído o se sabe, comporta algunos riesgos, como por ejemplo, ser etiquetado o estigmatizado como persona desleal, traidora, o poco fiable, y esto puede acarrear consecuencias tales como la expulsión de la comunidad o marginación dentro del grupo (Hallsworth y Young, 2008). Finalmente, cabe mencionar los datos que aportan los estudios empíricos realizados en el ámbito escolar, en los que se señala que el desinterés o apatía (Hektner y Swenson, 2012; Smith y Shu, 2000), la creencia de que las víctimas han de saber defenderse por sí mismas y los demás no han de intervenir (Hektner y Swenson, 2012), la tendencia a normalizar las situaciones de violencia entre los menores, como conductas aceptables y propias de la edad (Bauman et al., 2016; Pasupathi y McLean, 2010; Reis, 2007; Unnever y Cornell, 2004), y la falta de habilidad o conocimiento para identificar las situaciones de victimización (Hektner y Swenson, 2012; Oldenburg, van Duijn y Veenstra, 2016; Oldenburg et al., 2015) favorecen el silencio de profesionales e instituciones educativas ante la victimización infantojuvenil.

Las conductas de contar (voicing, telling) y callar (silencing) han sido estudiadas en relación a ciertos tipos de victimización infantojuvenil tales como los 
abusos sexuales y físicos (Crowley, 2007; Finkelhor et al., 1990; Frattaroli, 2006; Goodman-Brown et al., 2003; McElvaney et al., 2012), la violencia entre pares (Newman et al., 2001) o el bullying en el contexto escolar (Bauman et al., 2016; Hektner y Swenson, 2012). Ante estas experiencias se han hallado tasas de silencio que llegan al 70\% de las víctimas (Smith y Shu, 2000; Whitney y Smith, 1993). Sin embargo, y hasta donde conocemos, estas conductas no han sido estudiadas en relación a la EIV.

\subsection{Objetivo del estudio}

Teniendo en cuenta la falta de estudios centrados en las conductas de contar o callar ante la experiencias de EIV, el objetivo de este trabajo es conocer la prevalencia de las mismas sobre una variedad de experiencias de victimización, vinculadas a la exposición a violencia a lo largo de la vida, analizar su relación con variables sociodemográficas relevantes (sexo, edad y país o procedencia) señaladas en la literatura (Bauman et al., 2016; Finkelhor et al., 1990; Smith, Madsen y Moody, 1999), e identificar a qué personas se dirigen los adolescentes del sistema de justicia juvenil de Cataluña, uno de los grupos más afectados por este tipo de victimización.

\section{Método}

\subsection{Muestra}

La muestra está compuesta por 101 adolescentes (82 chicos y 19 chicas) infractores en edades comprendidas entre los 14 y los 17 años $(M=16.08$ años; $D T=0.99)$. Los jóvenes participantes se encontraban cumpliendo medidas de justicia juvenil en tres centros cerrados $(77.2 \%)$ y cinco centros abiertos (22.8\%) de Barcelona, y fueron seleccionados en base a los criterios de inclusión y exclusión (edad entre los 14 y los 17 años, sin dificultades para comprender el castellano o catalán y sin presencia de síntomas de desajuste que interfirieran con la respuesta a los instrumentos).

\subsection{Variables de estudio}

a. Características sociodemográficas. Los datos sociodemográficos se recogieron mediante un cuestionario creado ad hoc para este fin, incluyendo las variables 
relevantes para el estudio de la victimización infantojuvenil (Turner et al., 2007) y de las conductas de callar o contar (Oldenburg et al., 2016; Reitsema y Grietens, 2015; Smith y Shu, 2000; Smith et al., 1999; Unnever y Cornell, 2004). Los principales datos sociodemográficos de los participantes se detallan en la Tabla 1.

Tabla 1.

Características sociodemográficas de los participantes

\begin{tabular}{|c|c|c|c|c|c|c|c|}
\hline & \multicolumn{4}{|c|}{ Sexo } & \multirow{2}{*}{\multicolumn{2}{|c|}{ Total }} \\
\hline & & \multicolumn{2}{|c|}{ Chicos } & \multicolumn{2}{|c|}{ Chicas } & & \\
\hline & & $n$ & $\%$ & $n$ & $\%$ & $n$ & $\%$ \\
\hline \multirow[t]{2}{*}{ Edad } & $14-15$ & 19 & 23,20 & 8 & 42,10 & 27 & 26,70 \\
\hline & $16-17$ & 63 & 76,80 & 11 & 57,90 & 74 & 73,30 \\
\hline \multirow[t]{4}{*}{ País de origen } & España & 30 & 36,60 & 16 & 84,20 & 46 & 45,50 \\
\hline & Resto de Europa & 2 & 2,40 & 0 & 0,00 & 2 & 2,00 \\
\hline & Centro y Sudamérica & 21 & 25,60 & 2 & 10,50 & 23 & 22,80 \\
\hline & África & 29 & 35,40 & 1 & 5,30 & 30 & 29,70 \\
\hline \multirow{4}{*}{$\begin{array}{l}\text { Nivel } \\
\text { socioeconómico }\end{array}$} & Bajo & 39 & 56,50 & 4 & 25,00 & 43 & 50,60 \\
\hline & Medio-bajo & 18 & 26,10 & 2 & 12,50 & 20 & 23,50 \\
\hline & Medio & 9 & 13,00 & 6 & 37,50 & 15 & 17,60 \\
\hline & Medio-alto & 3 & 4,30 & 4 & 25,00 & 7 & 8,20 \\
\hline \multirow[t]{2}{*}{ Tipo de medida } & En centro cerrado & 16 & 19,50 & 7 & 36,80 & 23 & 22,80 \\
\hline & En centro abierto & 66 & 80,50 & 12 & 63,20 & 78 & 77,20 \\
\hline
\end{tabular}

b. Victimización por exposición indirecta o ser testigo de violencia (EIV). El instrumento utilizado para medir la prevalencia de la EIV fue el Juvenile Victimization Questionnaire (JVQ; Finkelhor et al., 2005) versión autoinforme, traducido al castellano y catalán, y adaptado a nuestro contexto cultural (Pereda, Gallardo-Pujol y Guilera, 2016) por el Grupo de Investigación en Victimización Infantil y Adolescente (GReVIA) de la Universidad de Barcelona. El JVQ comprende 36 tipos de victimización infantojuvenil agrupados en seis módulos, de los cuales en este estudio se utiliza el módulo de EIV que consta de nueve preguntas (W1 a W9) que abordan nueve tipos de experiencias de EIV en dos contextos: intrafamiliar (ítems W1 y W2) y comunitario (ítems W3 a W9), con opción de respuesta dicotómica (Sí-No). Si la respuesta es afirmativa, se continúa con las preguntas de seguimiento. En caso contrario, se continúa con el siguiente tipo de EIV. 
c. Contar o callar y a quién se cuenta. Se mide mediante la pregunta "¿Contaste a alguien lo que había ocurrido?", que se enuncia idénticamente para cada uno de los nueve tipos de EIV, y ofrece ocho opciones de respuesta múltiple (a nadie, a padres o tutores, otros familiares adultos, familiares menores, amigos, profesores/ entrenadores/ médicos, conocidos y pareja). Estas categorías no son mutuamente excluyentes, por lo cual cada participante puede emitir más de una respuesta por pregunta, excepto la categoría de respuesta "no conté a nadie", que sí lo es. La opción de respuesta "a la pareja" fue excluida del análisis por no haber sido emitida en ningún caso.

\subsection{Procedimiento}

La investigación fue aprobada por el consejo de investigación de la Universidad de Barcelona (IRB00003099) y se llevó a cabo según los principios éticos de la Declaración de Helsinki (World Medical Association, 2008), y del Código ético del Colegio Oficial de Psicólogos (COPC, 1989). Los jóvenes participantes y sus tutores firmaron previamente un consentimiento informado. Las entrevistas se realizaron individualmente según los principios éticos propuestos por UNICEF (2012) por entrevistadores entrenados en recolección de datos sobre violencia infantil.

\subsection{Análisis estadísticos}

Los datos se analizaron con el programa estadístico SPSS versión 22. Se calcularon las tasas globales de victimización, por contexto o ámbito de la violencia (familiar y comunitario) y por tipo de experiencia de EIV a lo largo de la vida. Entre los adolescentes victimizados, se calcularon los porcentajes de casos que indicaron cada opción de respuesta relativa a la conducta de silenciar o contar (i.e., a nadie, a padres o tutores, otros familiares adultos, familiares menores, amigos, profesores/entrenadores/ médicos, y conocidos) para cada tipo de EIV. Se calcularon luego las tasas de silencio o de haber contado a alguien la experiencia sufrida para cada uno de los nueve tipos de EIV, distribuyendo los casos por sexo, edad y procedencia, y se analizó la asociación entre estas variables mediante el estadístico Chi cuadrado de Pearson $\left(\chi^{2}\right.$, IC: $95 \%$ y $p<$ $0.05)$. 


\section{Resultados}

\section{Tasas de victimización por exposición indirecta a violencia}

La tasa de victimización global en el ámbito familiar es del 43.6\%, mientras que en el ámbito comunitario la tasa global es del 95\%. Las tasas más altas de victimización por EIV corresponden a experiencias del ámbito comunitario, alcanzándose el $82.2 \%$ en las agresiones con arma (W3) y el $81.2 \%$ en las agresiones sin arma (W4). En el ámbito familiar las tasas oscilan entre el $23.8 \%$ en las experiencias de violencia de los padres hacia otros hijos/as (W2) y el $28.7 \%$ de los progenitores entre sí (W1). Estos resultados se detallan en la Tabla 2.

\section{Contar o callar y a quién se cuenta}

Las tasas de silencio (no contar a nadie) y de contar a alguien las experiencias de EIV, detalladas en la Tabla 2, indican que la conducta más frecuente en los adolescentes de la muestra es no contarlas a alguien, con porcentajes del $75 \%$ en experiencias de violencia de los padres sobre los demás hijos (W2) y del 69\% de los padres entre sí (W1), ambas del ámbito intrafamiliar. En el ámbito comunitario, la tasa de silencio más alta se alcanza en la agresión sin arma (W4: 57.5\%) y, en segundo lugar, en el asesinato de un familiar o amigo (W6: 51.6\%).

En cuanto a los jóvenes que cuentan sus experiencias de EIV intrafamiliar, la mayoría se dirige, si se trata de violencia entre los padres (W1), en primer lugar, a otro miembro adulto de la familia (13.8\%) o a un profesor, entrenador o médico (10.3\%). Pero si se trata de violencia de los padres, madres o tutores hacia otro hijo o hija (W2), lo cuentan principalmente al otro progenitor $(16.7 \%)$ o a otro familiar adulto $(8.3 \%)$. A los amigos les suelen contar, sobre todo, experiencias del ámbito comunitario tales como disturbios callejeros y tiroteos (W8: 45.2\%) y agresiones con armas (W3: 42\%). Las experiencias de violencia más graves, como el asesinato de un familiar o amigo o de otra persona no tan allegada, no las cuentan a nadie en la mayoría de los casos (W6: $51.6 \%$ y W7: $45.8 \%$ respectivamente), pero los que sí lo hacen, se dirigen a los padres (W6: $22.6 \%$, y W7: 33.3\% respectivamente) o a los amigos (W7: 25\% y W6: $22.6 \%$ respectivamente). 


\section{Tabla 2.}

Interlocutor al que se cuentan las experiencias de EIV, en función del tipo de EIV

\begin{tabular}{|c|c|c|c|c|c|c|c|c|c|c|}
\hline \multicolumn{4}{|c|}{ Exposición indirecta a violencia } & \multicolumn{7}{|c|}{ ¿A quién se lo contaron? } \\
\hline \multirow[t]{2}{*}{$\begin{array}{l}\text { Ámbito o contexto } \\
\text { de la violencia }\end{array}$} & \multirow[t]{2}{*}{ Tipo de experiencia } & \multicolumn{2}{|c|}{$\begin{array}{c}\text { Total } \\
\text { victimizados }\end{array}$} & \multirow{2}{*}{$\begin{array}{c}\text { A nadie } \\
\%\end{array}$} & \multirow{2}{*}{$\begin{array}{c}\text { Padre/ } \\
\text { madre }\end{array}$} & \multirow{2}{*}{$\begin{array}{c}\text { Familiar adulto } \\
\text { (mayor de } 18 \\
\text { años) } \\
\%\end{array}$} & \multirow{2}{*}{$\begin{array}{c}\text { Familiar } \\
\text { joven } \\
\text { (menor de } \\
18 \text { años) } \\
\%\end{array}$} & \multirow{2}{*}{$\begin{array}{c}\text { Amigo } \\
\text { s } \\
\%\end{array}$} & \multirow{2}{*}{$\begin{array}{c}\begin{array}{c}\text { Profesor/ } \\
\text { entrenador/ } \\
\text { médico }\end{array} \\
\%\end{array}$} & \multirow{2}{*}{$\begin{array}{c}\begin{array}{c}\text { Conocid } \\
\text { os }\end{array} \\
\%\end{array}$} \\
\hline & & & $(\%)$ & & & & & & & \\
\hline \multirow{2}{*}{ Familiar } & W1 Entre padres/tutores & & (28.7) & 69.0 & - & 13.8 & 3.4 & 6.9 & 10.3 & - \\
\hline & W2. De padres a hermanos & 24 & (23.8) & 75.0 & 16.7 & 8.3 & - & - & 4.2 & - \\
\hline \multirow{7}{*}{ Comunitario } & W3. Agresión con arma & 83 & $(82.2)$ & 45.7 & 14.8 & 2.5 & 1.2 & 42.0 & 1.2 & 2.5 \\
\hline & W4. Agresión sin arma & 82 & $(81.2)$ & 57.5 & 3.8 & - & 2.5 & 40.0 & 2.5 & 2.5 \\
\hline & W5. Robo en el domicilio & & $(17.8)$ & 50.0 & 27.8 & 11.1 & - & 27.8 & - & - \\
\hline & $\begin{array}{l}\text { W6. Asesinato } \\
\text { familiar/amigo }\end{array}$ & & $(30.7)$ & 51.6 & 22.6 & 16.1 & 12.9 & 22.6 & - & 3.2 \\
\hline & W7. Asesinato & 24 & $(24.0)^{*}$ & 45.8 & 33.3 & 12.5 & 4.2 & 25.0 & - & 4.2 \\
\hline & W8. Disturbios/tiroteos & 31 & $(30.7)$ & 22.6 & 29.0 & 12.9 & 9.7 & 45.2 & - & 3.2 \\
\hline & W9. Guerra & 1 & $(1.0)^{* *}$ & 100.0 & - & - & - & - & - & - \\
\hline
\end{tabular}

$N=101$

* Prevalencia estimada con $n=100$ (un valor perdido).

** $n=1$ (sólo un caso). 


\section{Características sociodemográficas}

Los resultados relativos a la asociación entre las variables sociodemográficas y la conducta de contar o no las experiencias de EIV están detallados en la Tabla 3. En cuanto al sexo, los análisis realizados indican que no existen asociaciones estadísticamente significativas en el comportamiento de los chicos y las chicas en ninguna de las experiencias de EIV estudiadas. Sin embargo, se observa que las tasas de silencio de las chicas son más altas que las de los chicos, tanto en el ámbito intrafamiliar (88.9\% frente al 68.6\% de los chicos) como en el ámbito comunitario (31.3\% frente al $25 \%$ de los chicos). Las únicas experiencias en las que los chicos callan más que las chicas son las agresiones sin arma (W4: $58 \%$ de los chicos frente al $46.2 \%$ de las chicas) y el asesinato de un familiar o amigo (W6: 50\% de los chicos frente al 40\% de las chicas), ambas del ámbito comunitario. Las experiencias que más callan los chicos corresponden al ámbito intrafamiliar, en primer lugar la violencia de los padres hacia otros hijos (W2: 71.4\%) y luego de los padres entre sí (W1: 65.2\%).

Tampoco se encontraron asociaciones estadísticamente significativas entre el grupo de edad y la conducta de callar o contar. Se observa que los adolescentes mayores (16 y 17 años) callan las experiencias de EIV intrafamiliar con un porcentaje de silencio más alto que el del grupo de menor edad (78.6\% y $62.5 \%$ respectivamente). En cambio, son los más jóvenes quienes más frecuentemente callan sus experiencias de EIV comunitaria (36\% frente al $22.5 \%$ de los mayores) guardando, sobre todo, silencio sobre experiencias de violencia de los padres hacia otros hijos (W2: 75\%), asesinato de un familiar o amigo (W6: 71.4\%) y asesinato de alguien no allegado o robo en el domicilio familiar (W6 y W5: 66.7\% ambas por igual). Los adolescentes mayores callan, sobre todo, las experiencias del ámbito intrafamiliar: tales como la violencia entre los padres (W1: 82.4\%) y de los padres hacia los hermanos (W2: 75\%).

La comparación entre los jóvenes españoles y los extranjeros en cuanto a la conducta de callar o contar, tampoco ha proporcionado asociaciones estadísticamente significativas en ninguno de los ámbitos ni tipos de experiencias estudiados. Los españoles callan más que los extranjeros las experiencias de EIV intrafamiliares $(80 \%$ frente a $66.7 \%$ respectivamente), mientras que los extranjeros callan algo más que los españoles las del ámbito comunitario (28.8\% frente a $22.7 \%$ respectivamente). Españoles y extranjeros coinciden en callar, sobre todo, las EIV intrafamiliar, con 
porcentajes del $80 \%$ y $66.7 \%$ respectivamente. También coinciden en callar sobre las experiencias de violencia de padres o madres sobre hermanos o hermanas (W2: 77.8\% y $73.3 \%$ respectivamente) y la violencia de los padres entre sí (W1: 75\% y 64.7\% respectivamente). La experiencia de EIV comunitaria que más frecuentemente callan los españoles es el asesinato de un familiar o amigo (W6: 60\%), mientras que los extranjeros callan, sobre todo, las agresiones sin arma (W4: 64.3\%). 


\section{Tabla 3.}

Distribución de los jóvenes que no contaron sus experiencias de EIV en función del sexo, la edad y la procedencia

\begin{tabular}{|c|c|c|c|c|c|c|c|c|c|c|c|c|c|}
\hline \multirow{2}{*}{\multicolumn{2}{|c|}{$\begin{array}{c}\text { Tipo de experiencia de exposición } \\
\text { indirecta a violencia }\end{array}$}} & \multicolumn{4}{|c|}{ Sexo } & \multicolumn{4}{|c|}{ Edad } & \multicolumn{4}{|c|}{ Procedencia } \\
\hline & & \multirow{2}{*}{$\begin{array}{l}\text { Chicos } \\
n \quad(\%)\end{array}$} & \multirow{2}{*}{$\begin{array}{l}\text { Chicas } \\
n \quad(\%)\end{array}$} & \multirow[b]{2}{*}{$\chi^{2} \quad($ g.l. $)$} & \multirow[b]{2}{*}{$P$} & \multirow{2}{*}{$\begin{array}{l}\text { 14-15 } \\
n \quad(\%)\end{array}$} & \multirow{2}{*}{$\begin{array}{l}\text { 16-17 } \\
n \quad(\%)\end{array}$} & \multirow[b]{2}{*}{$\chi^{2} \quad$ (g.l.) } & \multirow[b]{2}{*}{$p$} & \multirow{2}{*}{$\begin{array}{l}\text { Españoles } \\
n \quad(\%)\end{array}$} & \multirow{2}{*}{$\begin{array}{c}\text { Extranjeros } \\
n \quad(\%)\end{array}$} & \multirow[b]{2}{*}{$\chi^{2}($ g.l. $)$} & \multirow[b]{2}{*}{$p$} \\
\hline & & & & & & & & & & & & & \\
\hline \multirow{3}{*}{ 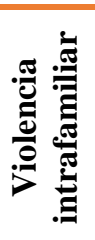 } & Total intra-familiar & $24(68.6)$ & $8(88.9)$ & $1.490(1)$ & 0.405 & $10(62.5)$ & $22(78.6)$ & $1.326(1)$ & 0.303 & $16(80.0)$ & $16(66.7)$ & $0.978(1)$ & 0.498 \\
\hline & W1. Entre padres/ tutores & $15(65.2)$ & $5(83.3)$ & $0.730(1)$ & 0.633 & $6(50.0)$ & $14(82.4)$ & $3.440(1)$ & 0.106 & $9 \quad(75.0)$ & $11(64.7)$ & $0.348(1)$ & 0.694 \\
\hline & W2. De padres a hermanos & $15(71.4)$ & $3(100)$ & $1.143(1)$ & 0.546 & $6(75.0)$ & $12(75.0)$ & $0.000(1)$ & 1.000 & $7 \quad(77.8)$ & $11(73.3)$ & $0.059(1)$ & 0.808 \\
\hline \multirow{7}{*}{ 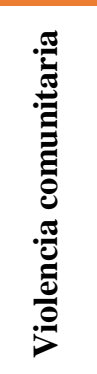 } & Total comunitaria & $20(25.0)$ & $5(31.3)$ & $0.270(1)$ & 0.755 & $9(36.0)$ & $16(22.5)$ & $1.740(1)$ & 0.197 & $10(22.7)$ & $15(28.8)$ & $0.463(1)$ & 0.641 \\
\hline & W3. Agresión con arma & $30(42.3)$ & $7(58.3)$ & $1.074(1)$ & 0.356 & $10(50.0)$ & $27(42.9)$ & $0.313(1)$ & 0.613 & $18(46.2)$ & $19(43.2)$ & $0.074(1)$ & 0.828 \\
\hline & W4. Agresión sin arma & $40(58.0)$ & $6(46.2)$ & $0.620(1)$ & 0.546 & $10(52.6)$ & $36(57.1)$ & $0.121(1)$ & 0.795 & $19(47.5)$ & $27(64.3)$ & $2.344(1)$ & 0.182 \\
\hline & W5. Robo en el domicilio & $6(50.0)$ & $3(50.0)$ & $0.000(1)$ & 1.000 & $2(66.7)$ & $7(46.7)$ & $0.400(1)$ & 1.000 & $5 \quad(45.5)$ & $4(57.1)$ & $0.234(1)$ & 1.000 \\
\hline & W6. Asesinato familiar o amigo & $13(50.0)$ & $2(40.0)$ & $0.168(1)$ & 1.000 & $5(71.4)$ & $10(41.7)$ & $1.922(1)$ & 0.220 & $6 \quad(60.0)$ & $9(42.9)$ & $0.797(1)$ & 0.458 \\
\hline & W7. Asesinato & $10 *(45.5)$ & $1(50.0)$ & $0.015(1)$ & 1.000 & $4(66.7)$ & $7(38.9)$ & $1.399(1)$ & 0.357 & $3(50.0)$ & $8(44.4)$ & $0.056(1)$ & 1.000 \\
\hline & W8. Disturbios/ tiroteos & $6 \quad(22.2)$ & $1(25.0)$ & $0.015(1)$ & 1.000 & $2(28.6)$ & $5(20.8)$ & $0.186(1)$ & 0.642 & $4(30.8)$ & $3(16.7)$ & $0.859(1)$ & 0.413 \\
\hline
\end{tabular}

$N=101 ; \chi^{2}$ : Chi cuadrado de Pearson; g.l.: grados de libertad; $p$ : significación estadística.

* Calculado con $n=100$ (un caso perdido). 


\section{Discusión}

El objetivo de este trabajo ha sido contribuir a la visibilidad de las múltiples experiencias de EIV a las que están expuestos, a lo largo de sus vidas, los adolescentes infractores, y observar si las cuentan o callan, y quiénes son sus interlocutores cuando deciden contarlas.

Los resultados obtenidos indican que callar es la respuesta más frecuente frente a la EIV, confirmándose que la cultura del silencio está vigente entre los adolescentes del sistema de justicia juvenil de Cataluña. No cuentan, en primer lugar, los episodios de violencia intrafamiliar, lo cual puede ser explicado por factores contextuales y relacionales, tales como un clima familiar que promueve el silencio y el secretismo, y los sentimientos de fidelidad hacia los progenitores y el grupo familiar (Reitsema y Grietens, 2015); o bien por factores individuales, como quedarse sin palabras a causa de las fuertes emociones (ira, tristeza, vergüenza, culpa) provocadas por la experiencia de EIV (Berger, 2004), por apatía (Grych y Fincham, 1990; Smith y Shu, 2000) o por haber adquirido insensibilidad por estar frecuentemente expuestos a esas experiencias (McCart et al., 2007).

En el ámbito comunitario, callan, en primer lugar, las agresiones sin arma, que son las experiencias más leves pero también de las más frecuentes entre estos jóvenes, por lo que podrían ser consideradas normales, carentes de interés y no merecedoras de ser contadas a nadie, dando soporte a lo que apunta Crowley (2007), en cuanto a que lo habitual, normal y corriente es lo que se calla, mientras que sí se cuentan los acontecimientos atípicos, definidos como eventos inesperados o traumáticos. El asesinato de un familiar o amigo, la experiencia más grave incluida en el cuestionario, es la segunda experiencia menos contada, lo cual es coherente con datos empíricos que demostraron que las emociones de gran intensidad (tristeza, miedo o rabia) activadas por experiencias de gran magnitud, predicen la conducta de silencio (Berger, 2004). Otras explicaciones del silencio frente a episodios muy intensos son querer mantenerse fiel al imperativo de mostrar fortaleza ante la adversidad, como signo de masculinidad (Fivush, 2010), o como muestra de autocontrol y valía personal, cualidades especialmente relevantes para los jóvenes infractores (Schalkwijk, et al., 2016).

En cuanto a la relación entre las conductas de contar o callar las experiencias de EIV y las características sociodemográficas estudiadas, nuestro estudio discrepa con 
otros realizados en el contexto escolar (bullying), en los que sexo y edad resultaron significativos en tanto que los chicos callan más que las chicas y los de mayor edad más que los menores (Bauman et al., 2016; Newman et al., 2001; Whitney y Smith, 1993), aunque estos mismos autores quienes recomiendan precaución a la hora de generalizar estos resultados a formas más graves de exposición a violencia (Newman et al., 2001).

La independencia entre las tres variables sociodemográficas estudiadas y la conducta de callar o contar en todos los tipos de EIV apunta a que convendría buscar explicación a la conducta de callar, en características individuales más complejas, tales como el trauma (Pennebaker y Beall, 1986), los procesos de la memoria, emociones y cogniciones de cada persona (Pasupathi y McLean, 2010), o bien, en las características del contexto social en que tiene lugar cada experiencia (Bauman et al., 2016; Dijkstra et al., 2008; Oldenburg et al., 2016; Reitsema y Grietens, 2015), observando las relaciones entre espectadores, víctimas directas, victimarios y demás participantes, y las normas (sociales, grupales) que rigen en el contexto concreto.

\subsection{Limitaciones}

Las limitaciones están, sobre todo, vinculadas a la composición de la muestra, puesto que la mayor parte de los jóvenes participantes cumplían medidas de internamiento (en centros cerrados), mientras que, por el contrario, en la población de justicia juvenil de Cataluña, la mayor parte de las medidas se cumplen en medio abierto, bajo supervisión de equipos profesionales, por lo que nuestros resultados no son generalizables a la población de menores infractores del sistema de justicia juvenil catalán. Además, por la baja proporción de chicas en la muestra, recomendamos prudencia a la hora de interpretar los resultados vinculados al sexo. Finalmente, cabe señalar que, en muchos casos, los participantes prefirieron no responder a la pregunta "a quién lo contaste", con lo cual la información que hemos podido recoger se ha visto reducida de manera considerable.

\subsection{Implicaciones}

El presente trabajo tiene el mérito de ser, dentro de lo que conocemos, el primer estudio llevado a cabo con un tipo de muestra escasamente empleada en estudios de victimización que proporciona datos empíricos sobre el comportamiento de contar a 
alguien o callar las experiencias de EIV, aportando elementos útiles para entender mejor los procesos y mecanismos que dificultan la detección, intervención y prevención de episodios de victimización que a menudo pasan inadvertidos para la sociedad (Craig et al., 2000; Oldenburg et al., 2016; UNICEF, 2014; Unnever y Cornell, 2004).

El empleo de un instrumento válido y fiable, adaptado a nuestro país (Pereda et al., 2016), que cada vez es más utilizado a nivel nacional e internacional, y también en muestras de justicia juvenil (Cuevas et al., 2007; DeHart y Moran, 2015; Pereda et al., 2015; Ruchkin et al., 2002), facilita la comparación de nuestros resultados con otros que en el futuro se realicen.

Nuestro estudio muestra que la prevalente conducta de guardar silencio ante las elevadas tasas de EIV de los jóvenes infractores dificulta que reciban la ayuda que podrían necesitar. Los interlocutores podrían contribuir a la recuperación saludable frente a estas experiencias, comentando y analizando con ellos los episodios de EIV y su papel frente a estas situaciones, ofreciéndoles visiones alternativas y propiciando cambios en sus actitudes, así como corrigiendo las creencias normalizadoras de la violencia, lo cual a su vez, puede redundar en la efectividad del tratamiento de reinserción que se esté ofreciendo al joven desde el sistema de justicia.

Cabe destacar que resulta indispensable y prioritario que no sólo los jóvenes expuestos, sino también los padres, tutores, profesionales e instituciones responsables de su educación, sean considerados objeto de intervención (Hektner y Swenson, 2012; Oldenburg et al., 2015). Esto implica ofrecerles información para que aprendan a identificar los episodios de violencia por EIV, y a fomentar en los jóvenes la empatía hacia las víctimas y el rechazo a la violencia. Asimismo conviene dotarlos de herramientas y técnicas útiles para la resolución pacífica de los conflictos, como la escucha activa, que fomenta la expresión de las emociones y permite la introducción de reflexiones e ideas para corregir las creencias distorsionadas en los jóvenes, preparándolos para afrontar posibles dificultades futuras y despertando la confianza en sí mismos (Vanistendael y Lecomte, 2002).

\section{Referencias}

Abram, K. M., Teplin, L. A., Charles, D. R., Longworth, S. L., Mcclelland, G. M., \& Dulcan, M. K. (2014). Posttraumatic Stress Disorder and trauma in youth in 
juvenile detention. JAMA Psychiatry, 61(4), 403-410. doi: 10.1001/archpsyc.61.4.403

Abram, K. M., Teplin, L. A., King, D. C., Longworth, S. L., Emanuel, K. M., Romero, E. G.,... Olson, N. D. (2013). PTSD, trauma, and comorbid psychiatric disorders in detained youth. Juvenile Justice Bulletin, (June), 1--14.

Akers, R. (1985). Deviant behavior: A social learning approach. Belmont, CA: Wadsworth.

Avakame, E. F. (1998). Intergenerational transmission of violence, self-control, and conjugal violence: A comparative analysis of physical violence and psychological aggression. Violence and Victims, 13(3), 301--316.

Bandura, A. (1977). Social learning theory. Englewood Cliffs, NJ: Prentice Hall.

Barker, E. D., Arseneault, L., Brendgen, M., Fontaine, N., \& Maughan, B. (2008). Joint development of bullying and victimization in adolescence: Relations to delinquency and self-harm. Journal of the American Academy of Child and Adolescent Psychiatry, 47(9), 1030-1038. doi: 10.1097/CHI.ObO13e31817eec98

Bauman, S., Meter, D. J., Nixon, C., \& Davis, S. (2016). Targets of peer mistreatment: Do they tell adults? What happens when they do? Teaching and Teacher Education, 57, 118-124. doi: 10.1016/j.tate.2016.03.013

Berger, C. R. (2004). Speechlessness. Causal attributions, emotional features and social consequences. Journal of Language and Social Psychology, 23(2), 147-179. doi: 10.1177/0261927X04263821

Besemer, S. (2011). Specialized versus versatile intergenerational transmission of violence: A new approach to studying intergenerational transmission from violent versus non-violent fathers: Latent class analysis. Journal of Quantitative Criminology, 28(2), 245-263. doi: 10.1007/s10940-011-9141-y

Black, D. S., Sussman, S., \& Unger, J. B. (2010). A further look at the intergenerational transmission of violence: witnessing interparental violence in emerging adulthood. Journal of Interpersonal Violence, 25(6), 1022-1042. doi: doi: $10.1177 / 0886260509340539$

Bohanek, J. G., Fivush, R., \& Walker, E. (2005). Memories of positive and negative emotional events. Applied Cognitive Psychology, 19(1), 51-66. doi: 10.1002/acp.1064

Brookmeyer, K. A., Henrich, C. C., \& Schwab-Stone, M. (2005). Adolescents who witness community violence: Can parent support and prosocial cognitions protect them from committing violence? Child Development, 76(4), 917-929. doi: 10.1111/j.1467-8624.2005.00886.x

Cisler, J. M., Begle, A. M., Amstadter, A. B., Resnick, H. S., Danielson, C. K., Saunders, B. E., \& Kilpatrick, D. G. (2012). Exposure to interpersonal violence and risk for PTSD, depression, delinquency, and binge drinking among adolescents: Data from the NSA-R. Journal of Traumatic Stress, 25(1), 33-40. doi: $10.1002 /$ jts. 21672

Covey, H. C., Menard, S., \& Franzese, R. J. (2013). Effects of adolescent physical abuse, exposure to neighborhood violence, and witnessing parental violence on adult socioeconomic status. Child Maltreatment, 18(2), 85-97. doi: $10.1177 / 1077559513477914$

Craig, W., Henderson, K., \& Murphy, J. (2000). Prospective teacher's attitudes toward bullying and victimization. Social Psychology International, 21(1), 5-21. doi: 10.1177/0143034300211001 
Crowley, M. S. (2007). Memories of childhood sexual abuse: narrative analyses of types, experiences, and processes of remembering. Journal of Interpersonal Violence, 22(9), 1095-1113. doi: 10.1177/0886260507302879

Cuevas, C. A., Turner, H. A., \& Ormrod, R. K. (2007). Juvenile Delinquency and Victimization. Journal of Interpersonal Violence, 22(12), 1581-1602. doi: $10.1177 / 0886260507306498$

Cyr, K., Chamberland, C., Clément, M. È., Lessard, G., Wemmers, J. H., Collin-Vézina, D., Gagné, M-H., \& Damant, D. (2013). Polyvictimization and victimization of children and youth: Results from a populational survey. Child Abuse and Neglect, 37(10), 814-20. doi: 10.1016/j.chiabu.2013.03.009

DeHart, D. D., \& Moran, R. (2015). Poly-victimization among girls in the justice system: Trajectories of risk and associations to juvenile offending. Violence Against Women, 21(3), 291-312. doi: 10.1177/1077801214568355

Dijkstra, J. K., Lindenberg, S., \& Veenstra, R. (2008). Beyond the class norm: Bullying behavior of popular adolescents and its relation to peer acceptance and rejection. Journal of Abnormal Child Psychology, 36(8), 1289-1299. doi: 10.1007/s10802008-9251-7

Egeland, B. (2010). Intergenerational transmission of criminal behavior: Understanding the effects of family criminality, interparental violence, maltreatment, and hostile parenting. University of Minnesota, Minnesota.

Eitle, D., \& Turner, R. J. (2002). Exposure to community violence and young adult crime: The effects of witnessing violence, traumatic victimization, and other stressful life events. Journal of Research in Crime and Delinquency, 39(2), 214237. doi: 10.1177/002242780203900204

Erdelja, S., Vokal, P., Bolfan, M., Erdelja, S. A., Begovac, B., \& Begovac, I. (2013). Delinquency in incarcerated male adolescents is associated with single parenthood, exposure to more violence at home and in the community, and poorer self-image. Croatian Medical Journal, 54(5), 460-8. doi: 10.3325/cmj.2013.54.460

Evans, S. E., Davies, C., \& DiLillo, D. (2008). Exposure to domestic violence: A metaanalysis of child and adolescent outcomes. Aggression and Violent Behavior, 13(2), 131-140. doi 10.1016/j.avb.2008.02.005

Finkelhor, D. (2007). Developmental victimology: The comprehensive study of childhood victimization. En R. C. Davies, A. J. Lurigio, \& S. Herman (Eds.), Victims of Crime (3rd. ed.) (pp. 9-34). Thousand Oaks CA: Sage Publications.

Finkelhor, D., Hamby, S. L., Ormrod, R., \& Turner, H. (2005). The Juvenile Victimization Questionnaire: Reliability, validity, and national norms. Child Abuse and Neglect, 29(4), 383-412. doi: 10.1016/j.chiabu.2004.11.001

Finkelhor, D., Hotaling, G., Lewis, I. A., \& Smith, C. (1990). Sexual abuse in a national survey of adult men and women: Prevalence, characteristics, and risk factors. Child Abuse and Neglect, 14(1), 19-28. doi: 10.1016/0145-2134(90)90077-7

Fivush, R. (2010). Speaking silence: the social construction of silence in autobiographical and cultural narratives. Memory, 18(2), 88-98. doi: doi: 10.1080/09658210903029404

Forcadell, A. J., Camps, C., Rivarola, P., \& Pérez, J. (2004). Avaluació de la reincidència dels menors desinternats del Centre Educatiu L'Alzina. Barcelona: Centre d'Estudis Jurídics i Formació Especialitzada.

Frattaroli, J. (2006). Experimental disclosure and its moderators: A meta-analysis. Psychological Bulletin, 132(6), 823-865. doi: 10.1037/0033-2909.132.6.823 
Goodman-Brown, T. B., Edelstein, R. S., Goodman, G. S., Jones, D. P. H., \& Gordon, D. S. (2003). Why children tell: A model of children's disclosure of sexual abuse. Child Abuse and Neglect, 27(5), 525-540. doi: 10.1016/S0145-2134(03)00037-1

Grych, J. H., \& Fincham, F. D. (1990). Marital conflict and children's adjustment: A cognitive-contextual framework. Psychological Bulletin, 108(2), 267-290. doi: 10.1037/0033-2909.108.2.267

Hallsworth, S., \& Young, T. (2008). Crime and silence: "Death and life are in the power of the tongue" (Proverbs 18:21). Theoretical Criminology, 12(2), 131-152. doi: $10.1177 / 1362480608089237$

Hektner, J. M., \& Swenson, C. A. (2012). Links from teacher beliefs to peer victimization and bystander intervention: Tests of mediating processes. The Journal of Early Adolescence, 32(4), 516-536. doi: 10.1177/0272431611402502

Herrera, V. M., \& McCloskey, L. A. (2001). Gender differences in the risk for delinquency among youth exposed to family violence. Child Abuse \& Neglect, 25(8), 1037-51. doi: 10.1016/S0145-2134(01)00255-1

Holden, G. W. (2003). Children Exposed to Domestic Violence and Child Abuse: Terminology and Taxonomy. Clinical Child and Family Psychology Review, 6(3), 151-160. doi: 10.1023/A:1024906315255

Huang, C. C., Vikse, J. H., Lu, S., \& Yi, S. (2015). Children's exposure to intimate partner violence and early delinquency. Journal of Family Violence, 30(8), 953965. doi: 10.1007/s10896-015-9727-5

Kaufmann, G., Drevland, G. C. B., Wessel, E., Overskeid, G., \& Magnussen, S. (2003). The importance of being earnest: Displayed emotions and witness credibility. Applied Cognitive Psychology, 17(1), 21-34. doi: 10.1002/acp.842

Kiewitz, C., Restubog, S. L. D., Shoss, M. K., Garcia, P. R. J. M., \& Tang, R. L. (2016). Suffering in silence: Investigating the role of fear in the relationship between abusive supervision and defensive silence. Journal of Applied Psychology, 101(5), 731-742. doi: 10.1037/apl0000074

Kitzmann, K. M., Gaylord, N. K., Holt, A. R., \& Kenny, E. D. (2003). Child witnesses to domestic violence: A meta-analytic review. Journal of Consulting and Clinical Psychology, 71(2), 339-352. doi: 10.1037/0022-006X.71.2.339

Lens, K. M. E., van Doorn, J., Lahlah, E., Pemberton, A., \& Bogaerts, S. (2016). Observers reactions to victim impact statements: A preliminary study into the affective and cognitive responses. International Review of Victimology, 22(1), 4553. doi: $10.1177 / 0269758015610851$

Loeber, R., \& Stouthamer-Loeber, M. (1998). Development of juvenile aggression and violence. American Psychologist, 53(2), 242-259. doi: 10.1037/0003066X.53.2.242

Mancho, R. (2012). Les agressions en contextos tancats de Justícia Juvenil. Barcelona: Centre d 'Estudis Jurídics i Formació Especialitzada.

Martín Solbes, V. M. (2008). Estudio socioeducativo de los jóvenes internados en las prisiones andaluzas. Víctor Manuel Martín Solbes. Revista Española de Investigación Criminológica, 6, 1-25.

Martínez-Catena, A., \& Redondo, S. (2013). Carreras delictivas juveniles y tratamiento. Zerbitzuan, (54), 171-183.

McCart, M. R., Smith, D. W., Saunders, B. E., Kilpatrick, D. G., Resnick, H., \& Ruggiero, K. J. (2007). Do urban adolescents become desensitized to community violence? Data from a national survey. American Journal of Orthopsychiatry, 
77(3), 434-442. doi: 10.1037/0002-9432.77.3.434

McElvaney, R., Greene, S., \& Hogan, D. (2012). Containing the secret of child sexual abuse. Journal of Interpersonal Violence, 27(6), 1155-75. doi: $10.1177 / 0886260511424503$

Menard, S., Covey, H. C., \& Franzese, R. J. (2015). Adolescent exposure to violence and adult illicit drug use. Child Abuse and Neglect, 42, 30-39. doi: 10.1016/j.chiabu.2015.01.006

Mrug, S., Madan, A., Cook, E. W., \& Wright, R. A. (2015). Emotional and Physiological Desensitization to Real-Life and Movie Violence. Journal of Youth and Adolescence, 44(5), 1092-1108. doi: 10.1007/s10964-014-0202-z

Newman, R. S., Murray, B., \& Lussier, C. (2001). Confrontation with aggressive peers at school: Students' reluctance to seek help from the teacher. Journal of Educational Psychology, 93(2), 398-410. doi: 10.1037/0022-0663.93.2.398

Oldenburg, B., Bosman, R., \& Veenstra, R. (2016). Are elementary school teachers prepared to tackle bullying? A pilot study. School Psychology International, 37(1), 64-72. doi: 10.1177/0143034315623324

Oldenburg, B., van Duijn, M., Sentse, M., Huitsing, G., van der Ploeg, R., Salmivalli, C., \& Veenstra, R. (2015). Teacher characteristics and peer victimization in elementary schools: A classroom-level perspective. Journal of Abnormal Child Psychology, 43, 33-44. doi: 10.1007/s10802-013-9847-4

Oriol Granado, X. (2013). Jóvenes delincuentes tutelados: Perfiles delictivos, desarrollo socioemocional y apego. Tesis doctoral. Universitat de LLeida, Lérida.

Pasupathi, M., \& Hoyt, T. (2009). The development of narrative identity in late adolescence and emergent adulthood: The continued importance of listeners. Developmental Psychology, 45(2), 558-574. doi: 10.1037/a0014431

Pasupathi, M., \& McLean, K. C. (2010). How silence affects memory, self, and society: foreword to the special issue. Memory, 18(2), 85-7. doi: 10.1080/09658210903225127

Pennebaker, J. W., \& Beall, S. K. (1986). Confronting a traumatic event: toward an understanding of inhibition and disease. Journal of Abnormal Psychology, 95(3), 274-281. doi: 10.1037/0021-843X.95.3.274

Pereda, N., Abad, J., \& Guilera, G. (2015). Victimization and polyvictimization of Spanish youth involved in juvenile justice. Journal of Interpersonal Violence, 129. doi: $10.1177 / 0886260515597440$

Pereda, N., Gallardo-Pujol, D., \& Guilera, G. (2016). Good practices in the assessment of victimization: The Spanish adaptation of the Juvenile Victimization Questionnaire from a causal indicators approach. Psychology of Violence. doi: 10.1037/vio0000075

Rechea, C., Fernández, E., \& Cuervo, A. (2008). Menores agresores en el ámbito familiar. Centro de Investigación en Criminología, 15, 1-80.

Reckdenwald, A., Mancini, C., \& Beauregard, E. (2013). The cycle of violence: Examining the impact of maltreatment early in life on adult offending. Violence and Victims, 28(3), 466-482. doi: 10.1891/0886-6708.VV-D-12-00054

Reis, J. (2007). Predictors of Middle School Aggression. Youth and Society, 38(3), 322-347. doi: 10.1007/s10935-006-0031-2

Reitsema, A. M., \& Grietens, H. (2015). Is anybody listening? The literature on the dialogical process of child sexual abuse disclosure reviewed. Trauma, Violence, \& Abuse, 17(3), 330-340. doi: 10.1177/1524838015584368 
Rousseau, S., Grietens, H., Vanderfaeillie, J., Hoppenbrouwers, K., Wiersema, J. R., Baetens, I., Vos, P., \& Van Leeuwen, K. (2014). The association between parenting behavior and somatization in adolescents explained by physiological responses in adolescents. International Journal of Psychophysiology, 93(2), 261266. doi: 10.1016/j.ijpsycho.2014.05.008

Ruchkin, V. V., Schwab-Stone, M., Koposov, R., Vermeiren, R., \& Steiner, H. (2002). Violence exposure, posttraumatic stress, and personality in juvenile delinquents. Journal of the American Academy of Child and Adolescent Psychiatry, 41(3), 322329. doi: 10.1097/00004583-200203000-00012

Schalkwijk, F., Stams, G. J., Stegge, H., Dekker, J., \& Peen, J. (2016). The conscience as a regulatory function: Empathy, shame, pride, guilt, and moral orientation in delinquent adolescents. International Journal of Offender Therapy and Comparative Criminology, 60(6), 675-693. doi: 10.1177/0306624X14561830

Sethi, D., Hughes, K., Bellis, M., Mitis, F., \& Racioppi, F. (2010). European report on preventing violence and knife crime among young people. Copenhagen: World Health Organisation (WHO).

Sethi, D. Bellis, M., Hughes, K., Gilbert, R., Mitis, F., \& Galea, G. (2013). European report on preventing child maltreatment European report on preventing child maltreatment. Copenhagen: World Health Organisation (WHO).

Smith, D. W., Letourneau, E. J., Saunders, B. E., Kilpatrick, D. G., Resnick, H. S., \& Best, C. L. (2000). Delay in disclosure of childhood rape: Results from a national survey. Child Abuse \& Neglect, 24(2), 273-287. doi: 10.1016/S01452134(99)00130-1

Smith, P. K., Madsen, K. C., \& Moody, J. C. (1999). What causes the age decline in reports of being bullied at school? Towards a developmental analysis of risks of being bullied. Educational Research, 41(3), 267-285. doi: 10.1080/0013188990410303

Smith, P. K., \& Shu, S. (2000). What good schools can do about bullying. Findings from a survey in English schools after a decade of research and action. Childhood, 7(2), 193-212. doi: 10.1177/0907568200007002005

Sorsoli, L. (2010). "I remember", "I thought", "I know I didn't say": Silence and memory in trauma narratives. Memory, 18(2), 129-141. doi: $10.1080 / 09658210903168046$

Staller, K. M., \& Nelson-Gardell, D. (2005). "A burden in your heart": Lessons of disclosure from female preadolescent and adolescent survivors of sexual abuse. Child Abuse and Neglect, 29(12), 1415-1432. doi: 10.1016/j.chiabu.2005.06.007

Thornberry, T. P., \& Krohn, M. D. (2004). Taking stock of delinquency. En H. B. Kaplan, A. E. Gottfried, \& A. W. Gottfried (Eds.). Longitudinal research in the social and behavioral sciences: An interdisciplinary series. New York: Kluwer Academic Publishers.

Turner, H. A., Finkelhor, D., \& Ormrod, R. (2007). Family structure variations in patterns and predictors of child victimization. The American Journal of Orthopsychiatry, 77(2), 282-295. doi: 10.1037/0002-9432.77.2.282

UNICEF. (2012). Ethical principles, dilemmas and risks in collecting data on violence against children: A review of available literature. New York: Statistics and Monitoring Section/Division of Policy and Strategy

UNICEF. (2014). Hidden in plain sight: A statistical analysis of violence against children. New York: Division of Data, Research and Policy 
Unnever, J. D., \& Cornell, D. G. (2004). Middle school victims of bullying: Who reports being bullied? Aggressive Behavior, 30(5), 373-388. doi: 10.1002/ab.20030

Van Domburgh, L., Loeber, R., Bezemer, D., Stallings, R., \& Stouthamer-Loeber, M. (2009). Childhood predictors of desistance and level of persistence in offending in early onset offenders. Journal of Abnormal Child Psychology, 37(7), 967-980. doi: 10.1007/s10802-009-9329-x

Vanistendael, S., \& Lecomte, J. (2002). La felicidad es posible: Despertar en niños maltratados la confianza en si mismos: construir la resiliencia. Barcelona: Gedisa.

Whitney, I., \& Smith, P. K. (1993). A survey of the nature and extent of bullying in junior/middle and secondary schools. Educational Reasearch, 35(1), 3-25. doi: $10.1080 / 0013188930350101$

Widom, C. S. (1989). The cycle of violence. Science, 244(4901), 160-166. doi: 10.1126/science. 2704995

Wolfe, D. A., Crooks, C. V., Lee, V., McIntyre-Smith, A., \& Jaffe, P. G. (2003). The effects of children's exposure to domestic violence: A meta-analysis and critique. Clinical Child and Family Psychology Review, 6(3), 171-187. doi: 10.1023/A: 1024910416164

World Health Organization (WHO). (2014). Investing in children: The European child and adolescent health strategy 2015-20120. Copenhagen.

World Medical Association. (2008). Ethical principles for medical research involving human subjects. Adopted by the 59th WMA General Assembly. Seoul, Korea.

Graciela Susanne es psicóloga por la Universidad de Ámsterdam (Países Bajos), mediadora judicial del Centro de Mediación de la Generalitat (CMDPC) y del Colegio de Psicólogos de Cataluña. Es profesora asociada del Grado en Criminología de la Universidad de Barcelona, Universidad Pompeu Fabra y Universitat Oberta de Catalunya, y ponente del Máster de Mediación del Ilustre Colegio de Abogados de Barcelona (ICAB) y del Consejo de los Ilustres Colegios de Abogados de Cataluña (CICAC). Actualmente desarrolla su tesis doctoral en el Grupo de Investigación en Victimización Infantil y Adolescente (GReVIA) sobre victimización por exposición indirecta a violencia en jóvenes infractores.

Noemí Pereda es doctora en Psicología por la Universidad de Barcelona y profesora titular de victimología en la misma universidad. Se ha especializado en la temática de la victimología del desarrollo, destacando por su trabajo en el ámbito del maltrato y el abuso sexual infantil. Ha colaborado con la Oficina Europea de la OMS en el estudio de la violencia infantojuvenil en España y participa en tareas de formación y asesoramiento en Save the Children, Fundación Vicki Bernadet o Fundación RANA. Dirige el Grupo de Investigación en Victimización Infantil y Adolescente (GReVIA) desde su creación, en 2009.

Georgina Guilera es doctora en Psicología por la Universidad de Barcelona y profesora en la Facultad de Psicología de esta misma universidad. Actualmente compagina la docencia en Psicometría con la investigación en la elaboración y adaptación de instrumentos de medida psicológica, las técnicas bibliométricas y meta-analíticas, y su 
aplicación en el ámbito de la salud mental y la victimología del desarrollo. Su aportación en estos ámbitos ha culminado con la publicación de sendos artículos en revistas científicas de reconocido prestigio internacional. Es miembro del Grupo de Investigación en Victimización Infantil y Adolescente (GReVIA) desde su creación. 